\title{
ANÁLISIS DE MORTALIDAD DE PACIENTES EN UNIDAD DE CUIDADOS INTENSIVOS EN UN HOSPITAL DEL DEPARTAMENTO DEL META, COLOMBIA
}

Norton Pérez Gutiérrez ${ }^{1}$

\section{Resumen}

El número de unidades de cuidado intensivo ha aumentado en Colombia en los últimos 15 años. Esto ha permitido el acceso de pacientes de áreas apartadas a este recurso. El manejo especializado disminuye la mortalidad, sin embargo, aún es elevada. El objetivo del estudio fue evaluar los factores asociados a la mortalidad en una UCI mixta de un hospital regional.

Se realizó un estudio de corte transversal con los datos de los pacientes que ingresaron en la unidad de cuidados intensivos del Hospital de Granada, Meta, Colombia; entre septiembre y diciembre de 2013.

Se revisaron los registros de 134 pacientes que ingresaron en el periodo de estudio. El 32\% de ellos falleció. Los factores asociados con la mortalidad fueron la gravedad, nivel de intervención terapéutica y necesidad de ventilación mecánica. Los pacientes críticos con puntajes altos de APACHE II y TISS-28 tienen mayor riesgo de fallecer, especialmente de forma temprana.

Palabras clave: cuidados críticos, enfermedad crítica, mortalidad, morbilidad, neumonía asociada al ventilador, Colombia.

${ }^{1}$ Médico Cirujano General. Medicina crítica y cuidado intensivo. Grupo de Investigación de Villavicencio - GRIVI Universidad Cooperativa de Colombia, sede Villavicencio. 


\section{ANALYSIS OF MORTALITY AND MORBIDITY OF PATIENTS IN AN INTENSIVE CARE UNIT IN A HOSPITAL IN THE DEPARTMENT OF META, COLOMBIA}

Norton Pérez Gutiérrez ${ }^{1}$

\section{Abstract}

The number of intensive care units has increased in Colombia the last 15 years. It has allowed that patients from remote areas access to this facility. Specialized treatment and care decreases mortality, however it is still high. The aim of the research was to evaluate mortality-associated factors in a mixed ICU in a regional hospital.

A cross-sectional study was conducted based on the data of patients admitted to the intensive care unit between September and December 2013 in the Hospital de Granada, Meta, Colombia.

One hundred and thirty-four records from patients admitted during the period of research were analyzed. Thirty-two percent of them died. Mortality-associated factors were severity, level of therapeutic intervention and need of mechanical ventilation. Critical care patients with high APACHE II and TISS-28 scores have higher risk to die in an early time. 


\section{ANÁLISE DA MORTALIDADE DOS PACIENTES EM UNIDADE DE TERAPIA INTENSIVA EM UM HOSPITAL DO DEPARTAMENTO DE META, COLÔMBIA}

Norton Pérez Gutiérrez ${ }^{1}$

\section{Resumo}

O número de unidades de terapia intensiva na Colômbia aumentou nos últimos 15 anos. Isso tem permitido o acesso a este recurso para os pacientes de áreas remotas. $\mathrm{O}$ tratamento especializado reduz a mortalidade, no entanto, continua a ser elevado. $\mathrm{O}$ objetivo da pesquisa foi avaliar os fatores associados à mortalidade em uma UTI mista em um hospital regional.

Foi realizado estudo transversal com dados de pacientes internados na unidade de terapia intensiva do Hospital de Granada, Meta, Colômbia, entre setembro e dezembro de 2013.

Registos de 134 pacientes que entraram no período do estudo, foram revisados. $32 \%$ deles morreram. Os fatores associados com a mortalidade foram gravidade, nível de intervenção terapêutica e a necessidade de ventilação mecânica Pacientes em estado crítico com altas pontuações APACHE II e TISS-28 têm um maior risco de morte, de uma forma precoce principalmente.

Palavras-chave: cuidados críticos, estado terminal, mortalidade, morbidez, pneumonia associada à ventilação mecânica, Colômbia. 


\section{Introducción}

Las unidades de cuidado intensivo han aumentado en los últimos años, lo que ha permitido la atención de pacientes en estado crítico en regiones apartadas de las grandes capitales (1). La atención de estos pacientes requiere un recurso humano especializado y altamente capacitado con dedicación exclusiva que permita su cuidado centralizado, a fin de disminuir la alta tasa de mortalidad asociada a estas patologías (2). Algunos autores han planteado la necesidad de la atención dedicada por intensivistas (3), aunque otros estudios de múltiples unidades no han encontrado diferencias en el resultado (4). El análisis de los indicadores en las unidades de cuidados intensivos permite conocer la incidencia de las patologías atendidas, así como los factores que influyen en la mortalidad y morbilidad asociada al cuidado, a fin de modificar los que son prevenibles. Entre ellos, se encuentra la evaluación de la gravedad de la población atendida con objeto de ajustar los desenlaces a estos. Uno de los puntajes más frecuentemente empleados es el APACHE II (5), aunque algunos autores han tratado de actualizarlo, o emplear otros puntajes con igual capacidad de discriminación para detectar los pacientes en riesgo de mortalidad (6). La intensidad de cuidado de enfermería es derivada de la gravedad del paciente y se ha asociado con la mortalidad en algunos estudios (6). Algunos estudios de la región han hecho búsqueda de los factores asociados a la mortalidad en unidades de cuidado intensivo (7-10), Orinoquia.
El objetivo del estudio fue evaluar los factores asociados con la mortalidad en una UCI mixta de un hospital regional. Las preguntas de investigación fueron: ¿En pacientes que ingresan a la unidad de cuidados intensivos, cuáles son los factores de riesgo principales para fallecer? La hipótesis del investigador fue: La mortalidad en la unidad de cuidados intensivos está asociada con la gravedad del paciente al ingreso.

\section{Metodología}

Tipo de estudio: corte transversal analítico.

Escenario: Hospital Departamental de Granada, Meta, Colombia.

Temporalidad: entre septiembre y diciembre de 2013.

Población de estudio: pacientes que ingresaron a cuidado intensivo durante el periodo de estudio.

Intervención: ninguna.

Desenlace: mortalidad.

Variables de estudio: demográficas, de morbilidad y gravedad.

Criterios de inclusión: se incluyeron los datos de los pacientes adultos ( $>18$ años) que ingresaron a la unidad de cuidados intensivos durante el periodo de estudio.

Criterios de exclusión: ninguno.

Selección de la muestra: la muestra fue seleccionada por método no probabi- 
lístico, no apareada de casos continuos por conveniencia de acuerdo con los criterios de inclusión y exclusión con todos los casos en el periodo de estudio. No se calculó tamaño muestral. La información fue extraída de los registros que se realizan a los pacientes du- rante su ingreso a la unidad, los cuales son consignados en una hoja de Excel. Esta base de datos contiene las variables estudiadas tanto categóricas como cuantitativas (edad, sexo, etc.), (Ver Tabla 1) y fue revisada y depurada para su análisis.

Tabla 1. Características de pacientes atendidos en la unidad de cuidados intensivos del Hospital de Granada entre septiembre y diciembre de 2013.

\begin{tabular}{lcc}
\hline \multicolumn{1}{c|}{ Total } & $\mathbf{1 3 4}$ & $\mathbf{1 0 0 , 0}$ \\
\hline Hombres & 87 & 64,9 \\
Tipo de aseguramiento & & \\
$\quad$ Contributivo & 39 & 29,1 \\
Subsidiado & 70 & 52,2 \\
SOAT & 6 & 4,5 \\
Vinculado & 19 & 14,2 \\
Diagnóstico & & \\
Cardiovascular & 18 & 13,4 \\
Gastrointestinal & 7 & 5,2 \\
GO & 7 & 5,2 \\
Hematológico & 1 & 0,7 \\
Neurológico & 42 & 31,3 \\
Otro infeccioso & 3 & 2,2 \\
Renal & 4 & 3,0 \\
Respiratorio & 52 & 38,8
\end{tabular}

Tipo ingreso

Cirugía programada

Cirugía urgencias

No cirugía

\section{Procedencia}

Hospitalización

Urgencias

Quirófanos

\section{Servicios}

Cirugía

GO 
Continuación Tabla 1. Características de pacientes atendidos en la unidad de cuidados intensivos del Hospital de Granada entre septiembre y diciembre de 2013.

\begin{tabular}{l|c|c}
\hline \multicolumn{1}{c|}{ Total } & $\mathbf{1 3 4}$ & $\mathbf{1 0 0 , 0}$ \\
\hline Neurocirugía & 43 & 32,1 \\
Otros & 3 & 2,2 \\
Destino al egreso & & \\
Fallece & 43 & 32,1 \\
Hospitalización & 80 & 59,7 \\
Remisión & 11 & 8,2 \\
Ventilados & 83 & 61,9 \\
NAV & 10 & 7,5 \\
Mortalidad temprana (<48 horas) & 21 & 15,7 \\
Mortalidad tardía & 22 & 16,4 \\
\hline
\end{tabular}

Fuente: unidad de cuidados intensivos.

Plan de análisis: el análisis estadístico se realizó en el programa SPSS versión 15. Se realizaron pruebas de estadística descriptiva en las que se evaluó la frecuencia y proporción de las variables categóricas, así como la tendencia central y dispersión de las variables continuas. En el análisis bivariado se compararon los resultados de las variables y la asociación de las variables independientes con los desenlaces con el fin de encontrar los factores asociados con la mortalidad y el tiempo de estancia en UCI. Las variables de estudio fueron:

- Variables independientes: sexo, edad, tipo de ingreso, diagnóstico, servicio de procedencia, especialidad, puntaje de gravedad APACHE II, puntaje de intervención TISS-28, ventilación mecánica, días de ventilación mecánica, neumonía asocia- da a la ventilación, asegurador, tipo de aseguramiento, destino.

- Variables dependientes: mortalidad, tipo de mortalidad, tiempo de estancia en UCI, neumonía asociada a la ventilación.

Por las características de las variables de desenlace se emplearon pruebas no paramétricas para la comparación de las variables continuas (U de Mann-Whitney) o de Kruskal Wallis, al igual que la prueba de Chi cuadrado, según corresponda por el tipo de variable de desenlace, y se estableció un valor de $\mathrm{p}<0,05$ como nivel de significancia.

Para el análisis multi-variado se empleó análisis de regresión logística de acuerdo a las características de las variables de desenlace. 


\section{Consideraciones éticas}

Debido a que se trató de un estudio retrospectivo que no involucra pacientes, sino las bases de datos de la entidad de salud, donde no se realizó ninguna intervención que pusiera en riesgo a los sujetos de investigación, no se requirió consentimiento informado a los pacientes de acuerdo a la resolución 8430 de 1993. Sin embargo, el protocolo de investigación fue estudiado y aprobado por el comité de investigación de la Universidad y posteriormente aprobado por la institución participante para su realización, publicación y respectiva divulgación de resultados.

\section{Resultados}

Durante el periodo de estudio 138 pacientes egresaron de la unidad de cuidados intensivos, entre los cuales se incluyeron 134 en el estudio debido a la falta de registros suficientes en 4 de ellos; la mayoría de los pacientes fueron hombres $(64,9 \%)$ con un promedio de edad de 54,8 $\pm 20,8$ años.

El tipo de aseguramiento de la mayoría de los pacientes fue del régimen subsidiado, seguido del régimen contributivo $(52,2$ y $29,1 \%$ respectivamente).

La causa de ingreso fue por diagnósticos de origen respiratorio y neurológico principalmente $(38,8$ y $31,3 \%)$ respectivamente, y un tipo de diagnóstico no quirúrgico en su mayoría $(72,4 \%)$, reflejado en pacientes principalmente del servicio de medicina interna y neurocirugía (50,8 y 32,1\%), (Ver Tabla 1 y Tabla 2).

Tabla 2. Características de las variables continuas de los pacientes atendidos en la unidad de cuidados intensivos del Hospital de Granada entre septiembre y diciembre de 2013.

\begin{tabular}{l|c|c|c|c|c|c|c}
\hline $\begin{array}{l}\text { Variables } \\
\text { Continuas }\end{array}$ & Promedio & DE & Mediana & Mínimo & Máximo & p25 & p75 \\
\hline Edad & 54,8 & 20,8 & 56 & 15 & 100 & 41 & 73 \\
Estancia & 4,7 & 5,5 & 2,5 & 0 & 33 & 1 & 6 \\
$\begin{array}{l}\text { Días de } \\
\text { ventilación }\end{array}$ & 4,7 & 5,2 & 3 & 1 & 30 & 1 & 7 \\
APACHE II & 19,8 & 9,4 & 18 & 1 & 48 & 14 & 25 \\
TISS-28 & 21,3 & 8,4 & 21 & 3 & 46 & 16 & 26 \\
\hline
\end{tabular}


La letalidad encontrada fue alta $(32,1 \%)$, con una gravedad alta que se evidencia en el puntaje de APACHE II $(19,8 \pm 9,4)$ y de intervención TISS-28 $(21,3 \pm 8,4)$. Cerca del $16 \%$ de los pacientes fallecieron de forma temprana ( $<48$ horas).

Hubo 637 días de estancia durante los 122 días del periodo de estudio, con un promedio de estancia de 4,7 \pm 5,5 días (Ver Tabla 2), un porcentaje ocupacio- nal del 55\%, un índice de rotación (giro de cama) de 3,5 y un promedio diario de 5,5 pacientes.

El $61,9 \%$ de los pacientes recibieron soporte ventilatorio con un promedio de 4,7 $\pm 5,2$ días de ventilación mecánica. El $7,5 \%$ de los pacientes $(12,1 \%$ de los ventilados) presentaron neumonía asociada a la ventilación mecánica (NAV) con una tasa de 25,8 NAV por 1.000 días de ventilación.

Tabla 3. Comparación de las variables continuas según el desenlace en pacientes hospitalizados en la unidad de cuidados intensivos en el Hospital Departamental de Granada, Meta, Colombia entre septiembre y diciembre de 2013.

\begin{tabular}{lccccc}
\hline \multicolumn{1}{c|}{ Variable } & $\begin{array}{c}\text { Fallecen } \\
\text { Temprano }\end{array}$ & $\begin{array}{c}\text { Fallecen } \\
\text { Tardío }\end{array}$ & Sobreviven & $\mathbf{p}$ \\
\hline $\mathbf{N}$ & 21 & 22 & 91 & \\
Edad & & & & \\
$\quad$ Promedio & 63,3 & 60,7 & 51,5 & $<0,05$ \\
$\quad$ DE & 19,4 & 21,7 & 20,2 & \\
$\quad$ Mediana & 65 & 58 & 52 & \\
Estancia & & & & $<0,05$ \\
$\quad$ Promedio & 1 & 6,2 & 5,3 & \\
$\quad$ DE & 0 & 4,5 & 5,8 & $<0,05$ \\
$\quad$ Mediana & 1 & 4,5 & 3 & \\
Días de ventilación & & & & \\
$\quad$ Promedio & 1 & 6,3 & 5,5 & $<0,05$ \\
DE & 0 & 4,2 & 6,0 & \\
Mediana & 1 & 5 & 4 & $<0,05$ \\
APACHE II & & & & \\
Promedio & 25,7 & 23,9 & 17,4 & \\
DE & 10,2 & 9,4 & 8,3 & \\
Mediana & 28 & 18,5 & 18 & \\
TISS-28 & & & & \\
Promedio & 27,2 & 25,7 & 18,8 & \\
DE & 8,4 & 8,2 & 7,3 & \\
Mediana & 27 & 25 & 20 & \\
\hline
\end{tabular}




\section{Bivariado}

Al comparar las variables independientes con los desenlaces se encontró que hubo mayor mortalidad en los pacientes con diagnóstico cardiovascular, neurológico, y respiratorio; los ingresados procedentes de hospitalización, de las especialidades de medicina interna, neurocirugía o los que tuvieron fueron sometidos a ventilación $(\mathrm{p}<0,05)$, (Ver Tabla 4).

Tabla 4. Comparación de las variables dependientes categóricas con la mortalidad en pacientes de unidad de cuidados intensivos del Hospital Departamental de Granada, Meta, Colombia entre septiembre y diciembre de 2013.

\begin{tabular}{l|c|c|c|c|c|c|c|c|c}
\hline Variable & $\mathbf{N}$ & Fallecen & $\%$ & $\begin{array}{c}\text { Sobrevi- } \\
\text { ven }\end{array}$ & $\%$ & OR & $\begin{array}{c}\text { IC } \\
\text { min }\end{array}$ & $\begin{array}{c}\text { IC } \\
\text { máx }\end{array}$ & $\mathbf{p}$ \\
\hline Total & 134
\end{tabular}

Sexo

$\begin{array}{llllllllll}\text { Hombres } & 87 & 30 & 34,5 & 57 & 65,5 & 1,377 & 0,6 & 2,884 & 0,419 \\ \text { Mujeres } & 47 & 13 & 27,7 & 34 & 72,3 & & & \end{array}$

\section{Tipo de}

aseguramiento

$\begin{array}{lccccccccc}\text { Contributivo } & 39 & 15 & 38,5 & 24 & 61,5 & & & & \\ \text { Subsidiado } & 70 & 22 & 31,4 & 48 & 68,6 & 1,216 & 0,731 & 2,023 & 0,4573 \\ \text { SOAT } & 6 & 2 & 33,3 & 4 & 66,7 & 0,926 & 0,203 & 7,683 & 1,0 \\ \text { Vinculado } & 19 & 4 & 21,1 & 15 & 78,9 & 2,344 & 0,652 & 8,411 & 0,1849\end{array}$

\section{Diagnóstico}

\begin{tabular}{|c|c|c|c|c|c|c|c|c|c|}
\hline Cardiovascular & 18 & 6 & 33,3 & 12 & 66,7 & 5,0 & 0,866 & 28,862 & 0,0565 \\
\hline Gastrointestinal & 7 & 1 & 14,3 & 6 & 85,7 & & & & \\
\hline $\mathrm{GO}$ & 7 & 0 & 0,0 & 7 & 100,0 & & & & \\
\hline Hematológico & 1 & 0 & 0,0 & 1 & 100,0 & & & & \\
\hline Neurológico & 42 & 15 & 35,7 & 27 & 64,3 & 5,556 & 1,139 & 27,099 & 0,022 \\
\hline $\begin{array}{l}\text { Otro } \\
\text { infeccioso }\end{array}$ & 3 & 0 & 0,0 & 3 & 100,0 & & & & \\
\hline
\end{tabular}


INVESTIGACIONES ANDINA No. 33 Vol. 18

Continuación Tabla 4. Comparación de las variables dependientes categóricas con la mortalidad en pacientes de unidad de cuidados intensivos del Hospital Departamental de Granada, Meta, Colombia entre septiembre y diciembre de 2013.

\begin{tabular}{|c|c|c|c|c|c|c|c|c|c|}
\hline Variable & $\mathbf{N}$ & Fallecen & $\%$ & $\begin{array}{c}\text { Sobrevi- } \\
\text { ven }\end{array}$ & $\%$ & OR & $\begin{array}{l}\mathrm{IC} \\
\min \end{array}$ & $\begin{array}{c}\text { IC } \\
\text { máx }\end{array}$ & $p$ \\
\hline Renal & 4 & 1 & 25,0 & 3 & 75,0 & & & & \\
\hline Respiratorio & 52 & 20 & 38,5 & 32 & 61,5 & 6,25 & 1,317 & 29,661 & 0,0115 \\
\hline Tipo ingreso & & 37 & & 79 & & & & & \\
\hline $\begin{array}{l}\text { Cirugía } \\
\text { programada }\end{array}$ & 14 & 1 & 7,1 & 13 & 92,9 & & & & \\
\hline $\begin{array}{l}\text { Cirugía } \\
\text { urgencias }\end{array}$ & 23 & 7 & 30,4 & 16 & 69,6 & 0,618 & 52,338 & 0,1234 & 0,618 \\
\hline No cirugía & 97 & 35 & 36,1 & 62 & 63,9 & 2,046 & 0,844 & 4,962 & 0,109 \\
\hline
\end{tabular}

\section{Procedencia}

$\begin{array}{lccccccccc}\text { Hospitalización } & 16 & 8 & 50,0 & 8 & 50,0 & 3,222 & 0,939 & 11,055 & 0,057 \\ \text { Urgencias } & 38 & 26 & 68,4 & 54 & 142,1 & 1,551 & 0,642 & 3,748 & 0,327 \\ \text { Quirófanos } & 80 & 9 & 11,3 & 29 & 36,3 & & & \end{array}$

\section{Servicios}

\begin{tabular}{lccccccccc} 
Cirugía & 14 & 1 & 7,1 & 13 & 92,9 & & & & \\
GO & 6 & 0 & 0,0 & 6 & 100,0 & & & & \\
$\begin{array}{l}\text { Medicina } \\
\text { interna }\end{array}$ & 68 & 27 & 39,7 & 41 & 60,3 & 14,488 & 1,843 & 113,908 & 0,0015 \\
Neurocirugía & 43 & 15 & 34,9 & 28 & 65,1 & 11,786 & 1,443 & 96,233 & 0,0058 \\
Otros & 3 & 0 & 0,0 & 3 & 100,0 & & & & \\
Ventilados & 83 & 40 & 48,2 & 44 & 53,0 & 14,884 & 4,293 & 51,606 & 0,00001 \\
NAV & 10 & 1 & 10,0 & 9 & 90,0 & 0,1 & 0,012 & 0,827 & 0,0112 \\
\hline
\end{tabular}


Se encontraron diferencias en las proporciones de mortalidad en el tipo de ingreso por cirugía programada $(\mathrm{p}=0,035)$, los pacientes de los servicios de cirugía $(\mathrm{p}=0,035)$, gineco-obstetricia ( $\mathrm{p}=0,085)$ y medicina interna $(\mathrm{p}=0,055)$. También se encontró significancia en otras variables como la NAV $(0,011)$ y la recepción de ventilación mecánica ( $\mathrm{p}=0,001)$, (Ver Tabla 4). Con los pacientes del servicio de medicina interna se encontró un valor cercano de significancia $(p=0,055)$.

Se encontró mayor estancia en UCI en las mujeres ( $\mathrm{p}=0,02$; ver Figura 1$)$ y los días de ventilación mecánica $(\mathrm{p}=0,03$; Ver Figura 2). No se encontraron diferencias en las variables continuas para la variable tipo de aseguramiento ( $p>0,05$; prueba de Kruskal-Wallis).

Al hacer las comparaciones estadísticas de las variables de estudio, se encontraron diferencias en la edad para las variables sistema diagnóstico, tipo de ingreso, procedencia, servicio $y$ destino en los pacientes ventilados $(\mathrm{p}<0,05)$. La diferencia de duración en la estancia en UCI y ventilación mecánica fue significativa en los pacientes ventilados y con neumonía asociada a la ventilación $(\mathrm{p}<0,05)$. También se encontraron diferencias en el APACHE II para las variables: sistema diagnóstico, servicio, pacientes ventilados, según el destino al egreso $(p<0,05)$. Otra diferencia encontrada para el puntaje de intensidad de cuidado (TISS-28) se evidenció para los pacientes ventilados y la variable destino al egreso $(p<0,05)$.
Los pacientes con diagnóstico cardiovascular tuvieron menos tiempo de estancia en UCI y mayor puntaje APACHE II. Los pacientes con diagnóstico neurológico tuvieron menor edad y días de ventilación mecánica. Los pacientes con diagnóstico respiratorio tuvieron menos edad, al igual que aquellos procedentes de hospitalización, del servicio de medicina interna y de neurocirugía. Estos últimos y los de medicina interna, también tuvieron mayor puntaje APACHE II.

\section{Desenlaces}

Al contrastar los pacientes que fallecieron o sobrevivieron se encontraron diferencias en las variables continuas como edad (Ver Figura 3), estancia en UCI (Ver Figura 4), puntaje de gravedad APACHE II (Ver Figura 5), puntaje de intervención terapéutica TISS-28 (Ver Figura 6), pero no en los días de ventilación mecánica. Estas diferencias se encontraron tanto en los pacientes con mortalidad temprana como tardía (mayor a 48 horas). De otro lado, en los paciente con mortalidad temprana se encontraron además diferencias en los días de ventilación mecánica $(\mathrm{p}<0,05$; prueba de Kruskal Wallis).

Hubo diferencias en la estancia en UCI según el destino al egreso, en los pacientes con neumonía asociada a la ventilación mecánica ( $\mathrm{p}<0,05$; prueba de Kruskal Wallis).

El puntaje APACHE II y el TISS-28 fueron mayores en los que fallecieron de forma tardía o temprana, al igual que el tiempo de estancia (Ver Tabla 3). 


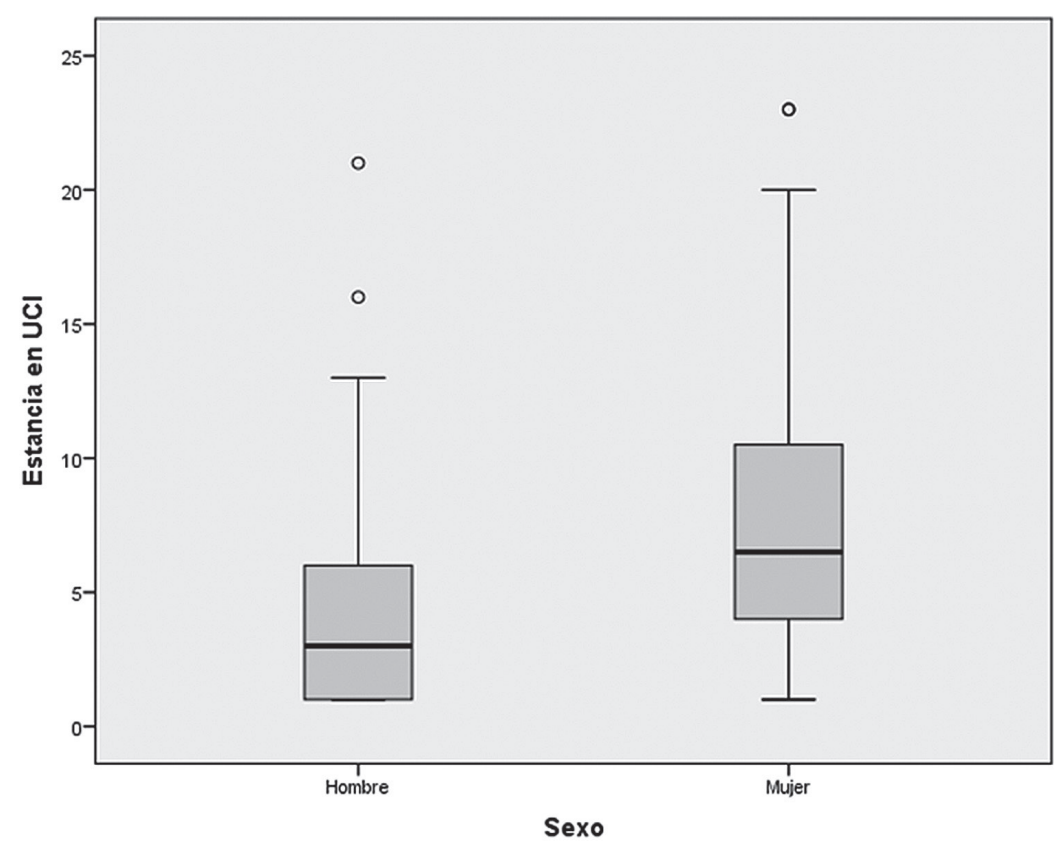

Figura 1. Comparación de la estancia en UCI por sexo. Hay mayor tiempo de estancia en UCI en las mujeres ( $\mathrm{p}=0,002$; prueba $U$ de Mann-Whitney).

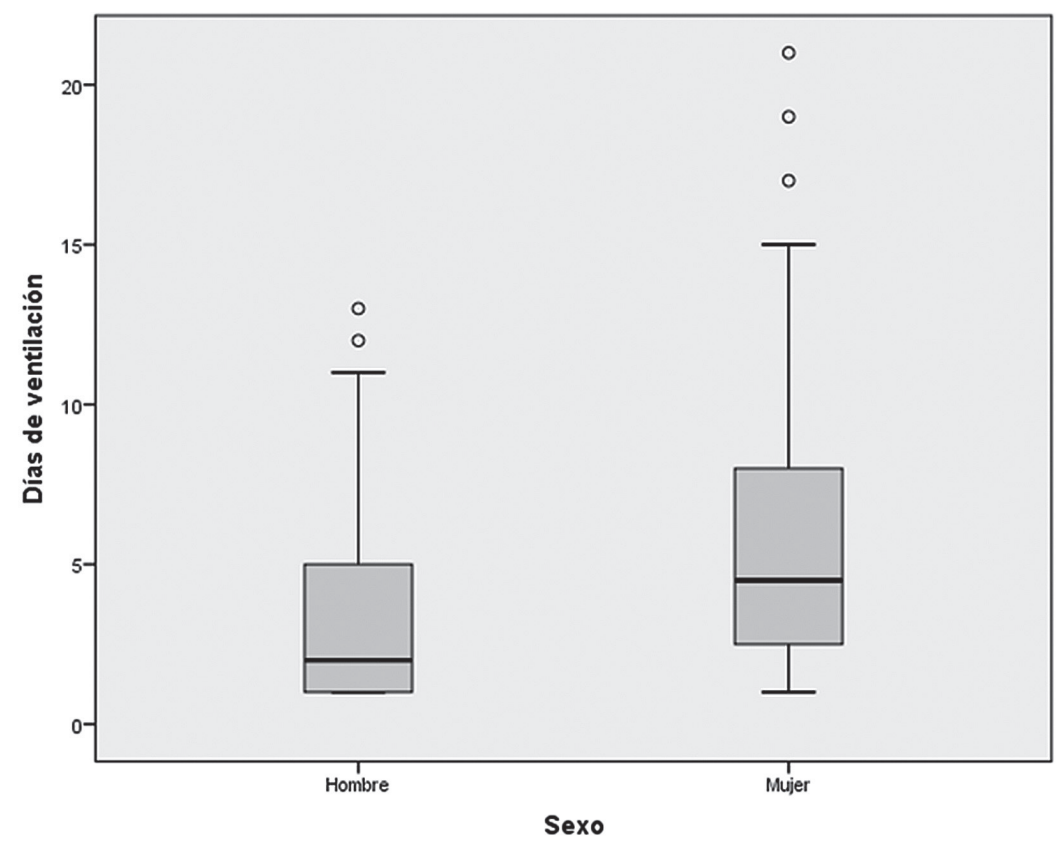
ventilación mecánica ( $\mathrm{p}=0,003$; prueba U de Mann-Whitney). 


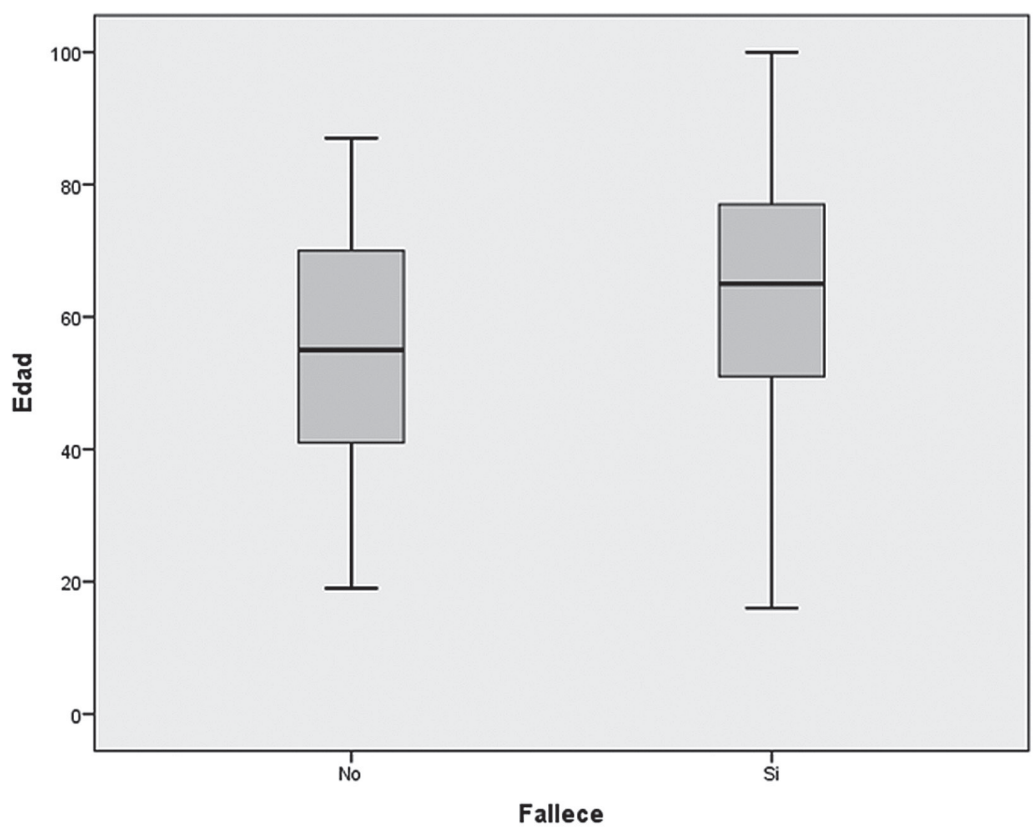

Figura 3. Comparación de la edad entre los pacientes que fallecieron o no en la unidad de cuidados intensivos de la Corporación Clínica Universidad Cooperativa de Colombia entre septiembre y diciembre de 2013 ( $\mathrm{p}<0,05$; prueba U de Mann-Whitney).

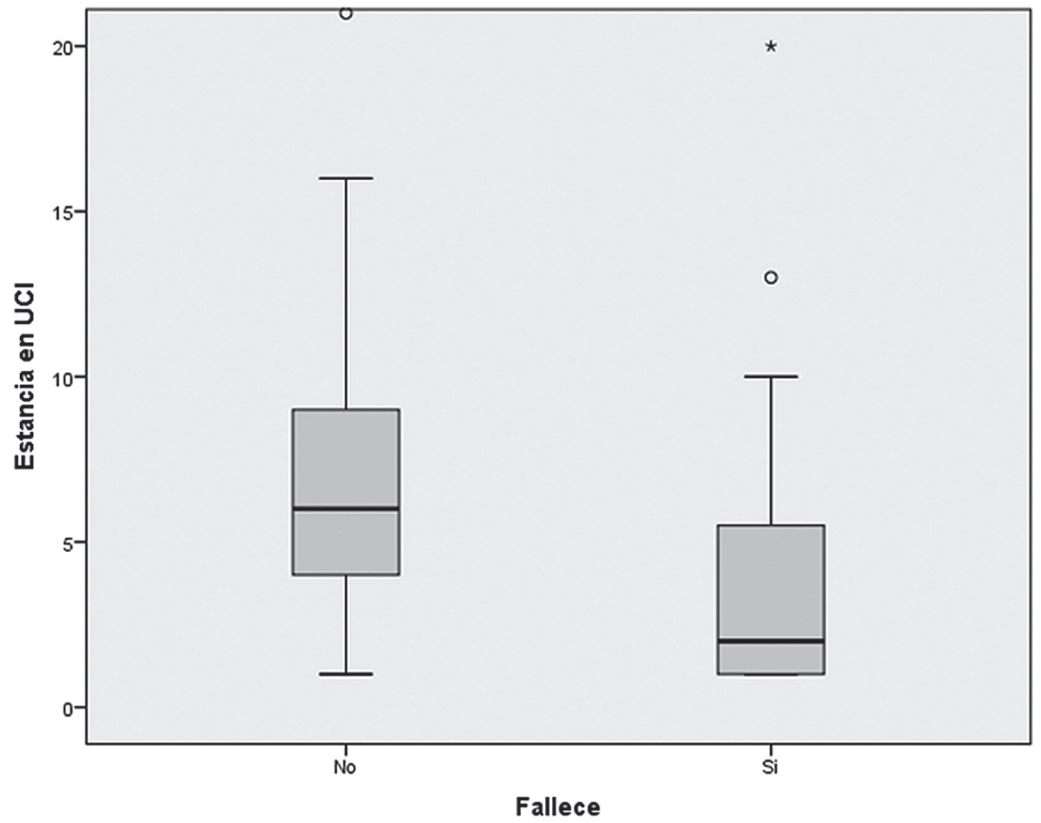

Figura 4. Comparación de la estancia en la UCI entre los pacientes que fallecieron o no en la unidad de cuidados intensivos de la Corporación Clínica Universidad Cooperativa de Colombia entre septiembre y diciembre de 2013 ( $\mathrm{p}<0,05$; prueba U de Mann-Whitney). 


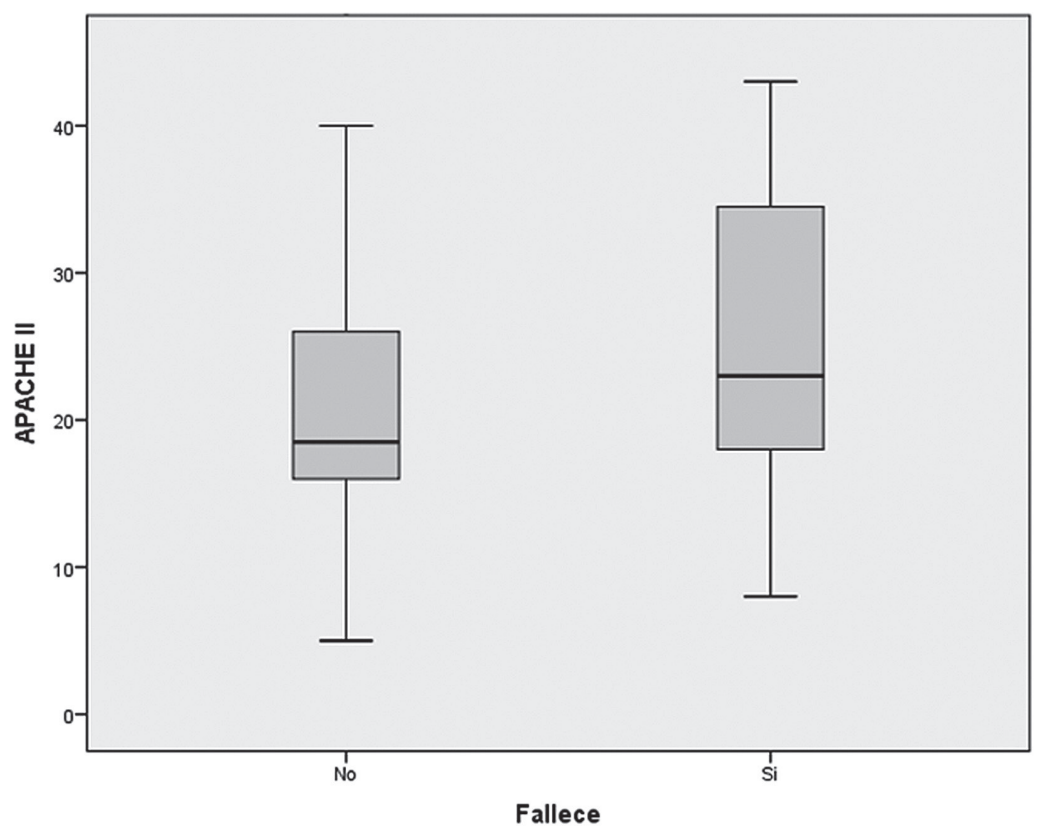

Figura 5. Comparación del puntaje de gravedad APACHE II de los pacientes que fallecieron o no en la unidad de cuidados intensivos de la Corporación Clínica Universidad Cooperativa de Colombia entre septiembre y diciembre de 2013 ( $\mathrm{p}<0,05$; prueba U de Mann-Whitney).

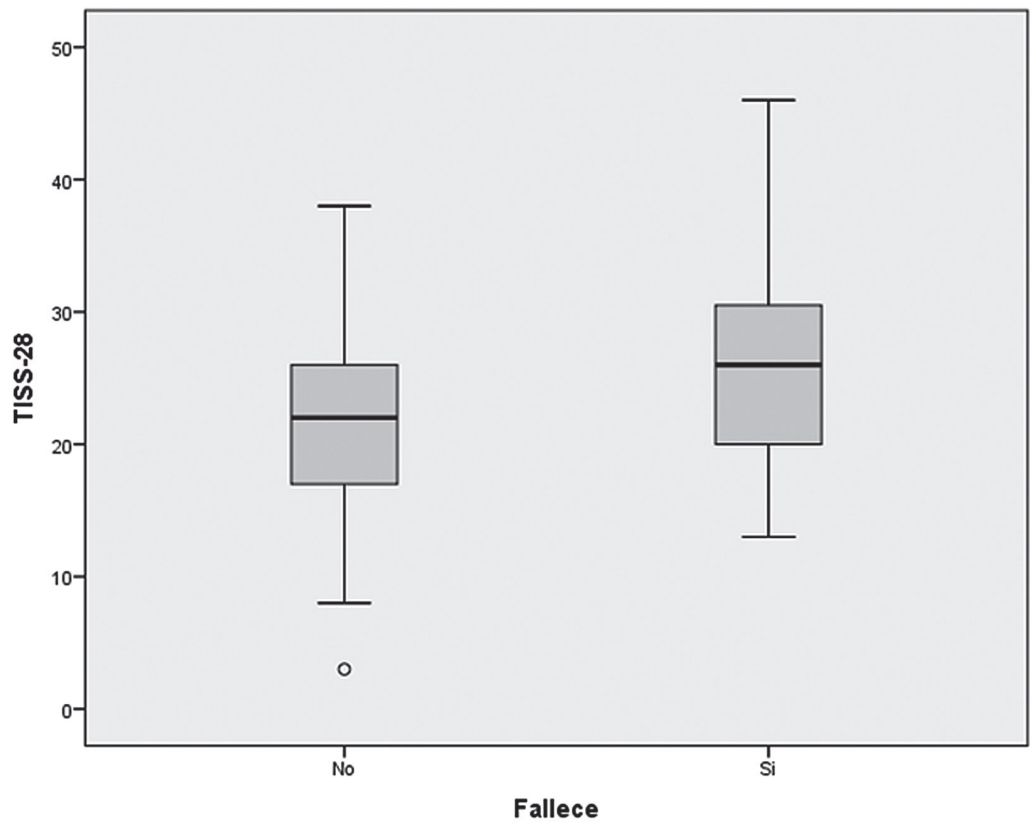

1618 Figura 6. Comparación del puntaje de intervención terapéutica TISS-28 entre los pacientes que fallecieron o no en la unidad de cuidados intensivos de la Corporación Clínica Universidad Cooperativa de Colombia entre septiembre y diciembre de 2013 ( $\mathrm{p}<0,05$; prueba U de Mann-Whitney). 
En el análisis de las variables categóricas se encontró mayor riesgo de mortalidad en los pacientes con diagnóstico cardiovascular, neurológico, respiratorio y aquellos procedentes de hospitalización o de los servicios de medicina interna o neurocirugía, al igual que aquellos que requirieron ventilación mecánica o desarrollaron neumonía asociada a esta $(\mathrm{p}<0,05)$.

\section{Análisis multivariado}

Las variables que no alcanzaron significancia, pero estuvieron igual $\mathrm{o}$ por debajo de $\mathrm{p}=0,1$ se incluyeron en el modelo de regresión logística.

Se construyó un modelo que permite predecir el riesgo de mortalidad del $54,5 \%$ cuando el paciente está ventilado, tiene un diagnóstico de origen respiratorio, procede de hospitalización $y$ cuando el servicio tratante fue medicina interna, mientras que la probabilidad de mortalidad sin ninguna de estas características es del 5,1\% (Ver Figura 7).

\section{Receiver operating characteristic}

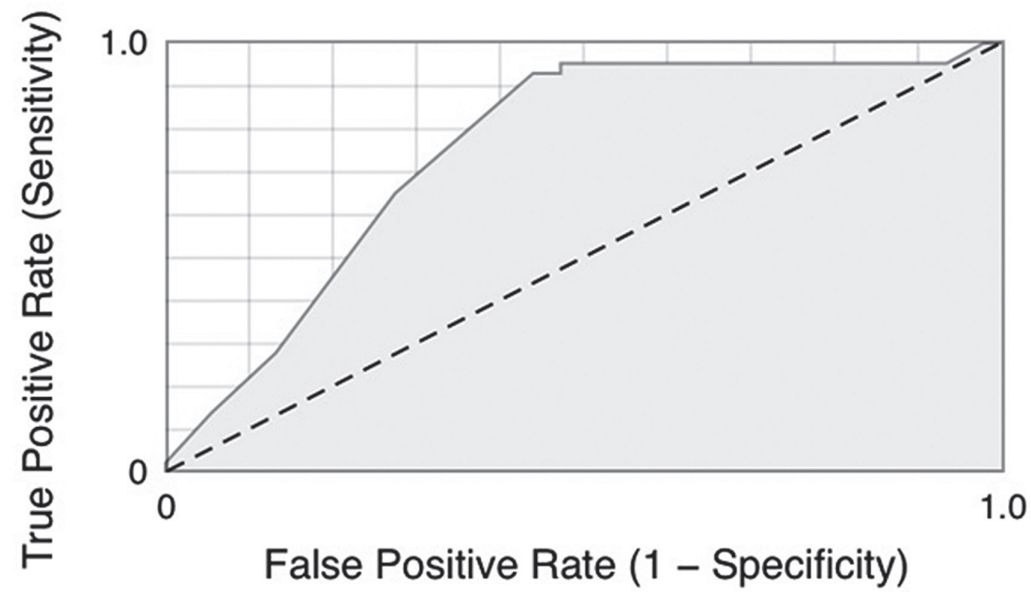

\section{Area Under Curve $=0.7530$}

Figura 7. Capacidad de predicción del modelo de predicción de mortalidad. Construido con el programa Wizard ${ }^{\circledR}$ versión 1,9,5 para Mac. 


\section{Discusión y conclusiones}

La unidad de cuidados intensivos es un área especializada en la atención de pacientes en situación crítica con alto riesgo de fallecimiento por la gravedad de la patología. Los avances tecnológicos en las áreas diagnósticas y terapéuticas han permitido allegar un mejor cuidado a esta población, pero aun así, los riesgos son altos (11). Varios modelos han sido desarrollados a fin de predecir cuáles son los subgrupos de enfermos con mayor riesgo de muerte a pesar del cuidado brindado, entre ellos se encuentran el puntaje de gravedad APACHE II y el puntaje de intervención terapéutica TISS-28 que son utilizados en la práctica diaria $(5,12-14)$.

El modelo de atención basado en un recurso humano especializado e interdisciplinario, con formación en el manejo del paciente crítico liderado por el curso de acción en la unidad a partir del desarrollo de guías de atención basadas en la evidencia, ha demostrado mejoría en los desenlaces clínicos, la eficacia y la eficiencia de los recursos, al igual que la fluidez en la comunicación y la toma de decisiones (2,15-18). Es por ello que la norma colombiana, en sus diferentes versiones, ha adoptado este modelo liderado por el intensivista $(19,20)$. Debido a ello, se requiere la realización del seguimiento de los indicadores de calidad en cada unidad, con el fin de reorientar los esfuerzos y modificar los factores susceptibles de cambio, entre ellos, la oportunidad $(21,22)$.

La UCI del Hospital de Granada es una unidad semi-cerrada, con 10 camas, que tiene como fundamento el manejo del paciente crítico por médicos especialistas (cirujanos e internistas) presenciales 24 horas al día, con el acompañamiento de un médico general (23). La relación de pacientes por enfermera es 2:1 (24). El cálculo del puntaje APACHE II es realizado por el médico a las 24 horas del ingreso. El cálculo del puntaje TISS-28 es realizado por la enfermera diariamente. Estas rutinas explican el hallazgo de puntajes bajos (7 registros), debido al fallecimiento de pacientes antes de cumplir las primeras 24 horas, pero que no permiten asociar esta muerte con la gravedad, lo cual pudiera significar que los puntajes podrían ser aún mayores. Estos valores fueron excluidos de los análisis para evitar hallazgos confusos. La mortalidad esperada para pacientes con APACHE II de 19 es alrededor del 29\%, lo cual fue similar a la encontrada en la unidad.

En el estudio se evidenció una alta proporción de mortalidad (32\%), del mismo modo que un alto puntaje de gravedad. Sólo el 16\% de las muertes se presentaron después de 48 horas (atribuible), de lo cual se podría concluir que el ingreso tardío u oportuno a la UCI podría estar asociado a la reducción o incremento de la mortalidad en los pacientes. Este factor amerita ser incluido en estudios posteriores, al igual que otros elementos como la procedencia del paciente en la medida en que entre más remota sea esta, más retrasará el ingreso oportuno a la unidad. Sin embargo, estas variables no fueron evaluadas en el estudio. Llama la atención el hallazgo relacionado con la baja utilización de las camas de UCI (de solo el 51\%), que contrasta con la tendencia nacional e internacional de un 
incremento en el uso de camas de cuidado crítico. También es particular que los pacientes procedentes de hospitalización tengan asociados altos puntajes en la escala de gravedad, de lo que se puede inferir demoras en la remisión a la UCI.

Los pacientes con patología cardiovascular, respiratoria y aquellos con patología neurológica tuvieron mayor riesgo de fallecer (Ver Tabla 4).También se encontró mayor riesgo de mortalidad en los pacientes procedentes de hospitalización, de la especialidades de medicina interna y neurocirugía, aquellos ventilados y los que desarrollaron NAV $(\mathrm{p}<0,05)$. Del mismo modo, se encontraron diferencias en las variables continuas, especialmente los puntajes de gravedad de estos subgrupos.

No se encontraron diferencias en el riesgo de mortalidad de acuerdo al tipo de aseguramiento. A pesar de estos hallazgos en el análisis bivariado, al ser incluidos en el análisis de regresión logística, solo se sostuvo las variable de gravedad. Adicionalmente, se encontraron diferencias significativas en las variables continuas, especialmente el puntaje de gravedad, de los pacientes con mortalidad temprana vs tardía, en contraste con los que sobrevivieron.

En un estudio mexicano, publicado en una revista española, se encontró una mortalidad en UCI del 25\%, pero la población atendida fue considerablemente de menor edad ( $47 \pm 17$ vs $54,8 \pm 20,8)$ y gravedad, aunque el método de evaluación fue diferente (SOFA y SAPS II vs APACHE II), (22). Otra publicación de una tesis doctoral de sociología, con una etnógrafa en la unidad de cuidados intensivos, describe otro tipo de pacientes muy diferente en un hospital de Barcelona en el año 2000, con 24.000 ingresos, mayormente entre 60-80 años (60\%), de los cuales el $1,77 \%$ ingresaron a UCI, con menor gravedad, también con predominancia de la patología respiratoria, un promedio de estancia de 15,2 días y una mortalidad del 25\% (25).

Para la construcción de un modelo explicativo se realizó un análisis de regresión logística que demostró el efecto del estado de gravedad del paciente en la mortalidad, que puede estar asociada a la demora por las grandes distancias en la región, previo a su ingreso a UCI. Ello podría explicar la alta proporción de mortalidad temprana, que ameritaría un análisis focalizado en ese aspecto en estudios futuros para evaluar si la mejor oportunidad de llegada a la UCI logra disminuir el desenlace fatal. La importancia del estudio radica en entender las causas de mortalidad alta a fin de establecer estrategias que lleven a mejorar la oportunidad en el ingreso a esta UCI y disminuir la mortalidad temprana o no atribuible (16\%).

La base de datos no contiene el seguimiento del paciente después de su salida de UCI, por lo cual no se pudo conocer la mortalidad hospitalaria a los 30 días. Esto es una limitante del estudio, al igual que el desconocimiento del municipio o ciudad de procedencia del paciente desde donde fuera remitido. Con esta información se podrían establecer los tiempos previos a su ingreso a UCI e incluso considerarlo como un posible factor a intervenir para mejorar las oportunida- 
des de acceso a la unidad y de este modo disminuir las tasas de mortalidad.

\section{Agradecimientos}

A la enfermera Paula Andrea Sánchez, por el registro juicioso en la base de datos que permitió este análisis.

\section{Declaración de conflicto de intereses}

El autor declara que no tiene conflictos de interés para la realización de este estudio.

\section{Autor de correspondencia}

Norton Pérez-Gutiérrez

Teléfono: +57 (86) 6729698

Correo electrónico:

norton.perez@hotmail.com

Dirección: Calle 36 \# 36 - 50

Villavicencio, Colombia

\section{Financiación}

Ninguna.

\section{Referencias}

1. Pérez Gutiérrez N, Rodríguez Darabos El. Talento humano en unidades de cuidado intensivo: adaptación de un modelo de estándares para Colombia basado en la evidencia científica. Acta Colomb Cuid Intens. 2015;15(2):80-102.

2. Hackner D. A primer: Staffing the ICU for performance, part 2: Refining the process with staff. ICU Dir. 2010 Jul 23;1(4):230-7.

3. Wilcox ME, Chong C a KY, Niven DJ, Rubenfeld GD, Rowan KM, Wunsch $\mathrm{H}$, et al. Do intensivist staffing patterns influence hospital mortality following ICU admission? A systematic review and meta-analyses. Crit Care Med. 2013;41(10):2253-74.

4. Costa DK, Wallace DJ, Kahn JM. The association between daytime intensivist context of other ICU organizational prac-

tices: A multicenter cohort study. Crit Care Med. 2015;43(11):2275-82.

5. Knaus WA, Drapper EA, Wagner DP, Zimmerman JE. APACHE II: A severity of disease classification system. Crit Care Med. 1985;13(10):818-29.

6. Efendijev I, Raj R, Skrifvars MB, Hoppu S, Reinikainen M. Increased need for interventions predicts mortality in the critically ill. Acta Anaesthesiol Scand. 2016;60(10):1415-24.

7. Oliveros Rodriguez $H$, Martinez Pacheco F, Lobelo Garcia R, Santrich $D$. Factores de riesgo determinantes de mortalidad postoperatoria en $\mathrm{UCl}$, en los pacientes quirúrgicos de alto riesgo. Rev Colomb Anestesiol. 2005;33(1):17-23.

8. Vasquez Jimenez LF, Castro Perez JA, Sara Ochoa JE. Factores de riesgo aso- 
ciados a mortalidad en pacientes sometidos a cirugía cardiaca. Estudio de cohorte prospectiva. Acta Colomb Cuid Intens. 2017;16:270-6.

9. Dennis RJ, Pérez A, Rowan K, Londoño D, Metcalfe A, Gómez C, et al. Factores asociados con la mortalidad hospitalaria en pacientes admitidos en cuidados intensivos en Colombia. Arch Bronconeumol. 2002;38(3):117-22.

10. Illera D, Rivera G, Orizco AE, Montenegro VM, Vidal CO. Perfil epidemiológico y factores de riesgo en pacientes de la unidad de cuidados intensivos, Hospital San José, Popayán. Rev Fac Ciencias la Salud Univ del Cauca. 2015;17(1):14-9.

11. Dueñas-Castell C, Ortiz Ruiz G. La atención médica en la unidad de cuidado intensivo en Colombia. Acta Colomb Cuid Intens. 2014;14(1):5-7.

12. Miranda RD, de Rijk A, Schaufeli W. Simplified Therapeutic Intervention Scoring System: The TISS-28 items - Results from a multicenter study. Crit Care Med. 1996;24:64-73.

13. Padilha KG, Sousa RM, Kimura M, Miyadahira AM, da Cruz DA, Vattimo M de $F$, et al. Nursing workload in intensive care units: a study using the Therapeutic Intervention Scoring System-28 (TISS-28). Intensive Crit Care Nurs. 2007;23(3):162-9.

14. Padilha KG, de Sousa RMC, Queijo AF, Mendes AM, Reis Miranda D. Nursing Activities Score in the intensive care unit: analysis of the related factors. Intensive Crit Care Nurs. 2008 Jun;24(3):197-204.

15. Dimick JB, Pronovost PJ, Heitmiller RF, Lipsett $P$ a. Intensive care unit physician staffing is associated with decreased length of stay, hospital cost, and complications after esophageal resection. Crit Care Med. 2001 May;29(4):753-8.

16. Ensminger SA, Morales IJ, Peters SG, Keegan MT, Finkielman JD, Lymp JF, et al. The hospital mortality of patients admitted to the ICU on weekends. Chest. 2004 Oct;126(4):1292-8.

17. Gutsche JT, Kohl B a. Who should care for intensive care unit patients? Crit Care Med. 2007 Mar;35(2 Suppl):S18-23.

18. Multz a S, Chalfin DB, Samson IM, Dantzker DR, Fein a M, Steinberg HN, et al. A "closed" medical intensive care unit (MICU) improves resource utilization when compared with an "open" MICU. Am J Resp Crit Care Med. 1998 May;157:1468-73.

19. Gaviria Uribe A. Por el cual se definen los procedimientos y condiciones que deben cumplir los Prestadores de Servicios de Salud para habilitar los servicios y se dictan otras disposiciones. Resolución número 1441 Colombia: Ministerio de Salud y Protección Social; 2013 p. 209.

20. Gaviria Uribe A. Por la cual se definen los procedimientos y condiciones de inscripción de los prestadores de servicios de salud y de habilitación de servicios de salud. Resolución 2003 Colombia: Ministerio de Salud y Protección Social; 2014 p. 225.

21. Martín Delgado MC, Blanco Varela J, Cabré Pericas L, Galdos Anuncibay P, Federico GV, editors. Indicadores de calidad en el enfermo crítico. 2a ed. Madrid: Sociedad Española de medicina intensiva, crítica y unidades coronarias; 2011. 188 p.

22. Alvarez-Maldonado $P$, Cueto-Robledo G, Ceron-Diaz U, Perez-Rosales A, Navarro-Reynoso F, Cicero-Sabido R. Indicadores de calidad en una unidad de cuidados intensivos respiratorios. Analisis inicial de la base de datos DEDUCIR. Med Intens. 2012 Oct;36(7):518-20.

23. Dara SI, Afessa B. Intensivist-to-bed ratio: association with outcomes in the medical ICU. Chest. 2005 Aug;128(2):567-72.

24. Haupt MT, Bekes CE, Brilli RJ, Carl LC, Gray AW, Jastremski MS, et al. Guidelines 
INVESTIGACIONES ANDINA No. 33 Vol. 18

on critical care services and personnel: Recommendations based on a system of categorization of three levels of care. Crit Care Med. 2003 Dec;31(11):2677-83.
25. Pallarés i Martí $A$. El mundo de las unidades de cuidados intensivos: la última frontera. Universitat Rovira I Virgili; 2003. 